\title{
Polypharmacy Drug Errors in the Elderly Peri- operative population; are we providing a disservice to an at-risk group?
}

\author{
Dr J Isherwood, Dr A Powell, Dr S Saxena
}

\section{Background}

There are 11.6 Mil people over the age of 65 in the UK, and by 2040 it is predicted that 1 in 4 UK residents will be over $65^{1}$. This age group will usually have a number of medical conditions and therefore will have been prescribed a variety of medications by their GP, and it is vital that these regular medications are continued in secondary care. In addition, the admission to hospital may often be as a result of an acute condition that necessitates the temporary alteration, or discontinuation of these drugs. Elderly patients are more vulnerable to the effects of some drugs, especially so in cases of renal impairment ${ }^{2}$. Changes must be communicated back to Primary Care in order to guarantee continuity.

\section{Aims}

- To ascertain whether regular medications were being effectively continued in the hospital setting.

- To investigate whether regular medications were altered in response to changes in eGFR.

\section{Materials and methods}

In this retrospective report of practice, the admissions of 34 patients over the age of 75 were investigated for the maintenance of their routine medication regimes while admitted and whether its continuation was recorded on discharge. Patients were selected at random from 3 surgical wards. Prescribed medicines were compared with reconciliation data from pharmacy, and collated against electronically recorded blood test results.

Positive Examples

84 year old female admitted following a fall, GFR dropped from 80 to below 60. Patient's regular NSAIDs stopped the next day.

77 year old male admitting with nausea and vomiting, AKI identified upon admission. Patient's regular Atorvastatin stopped and crossed out for next 3 days on the drug chart.

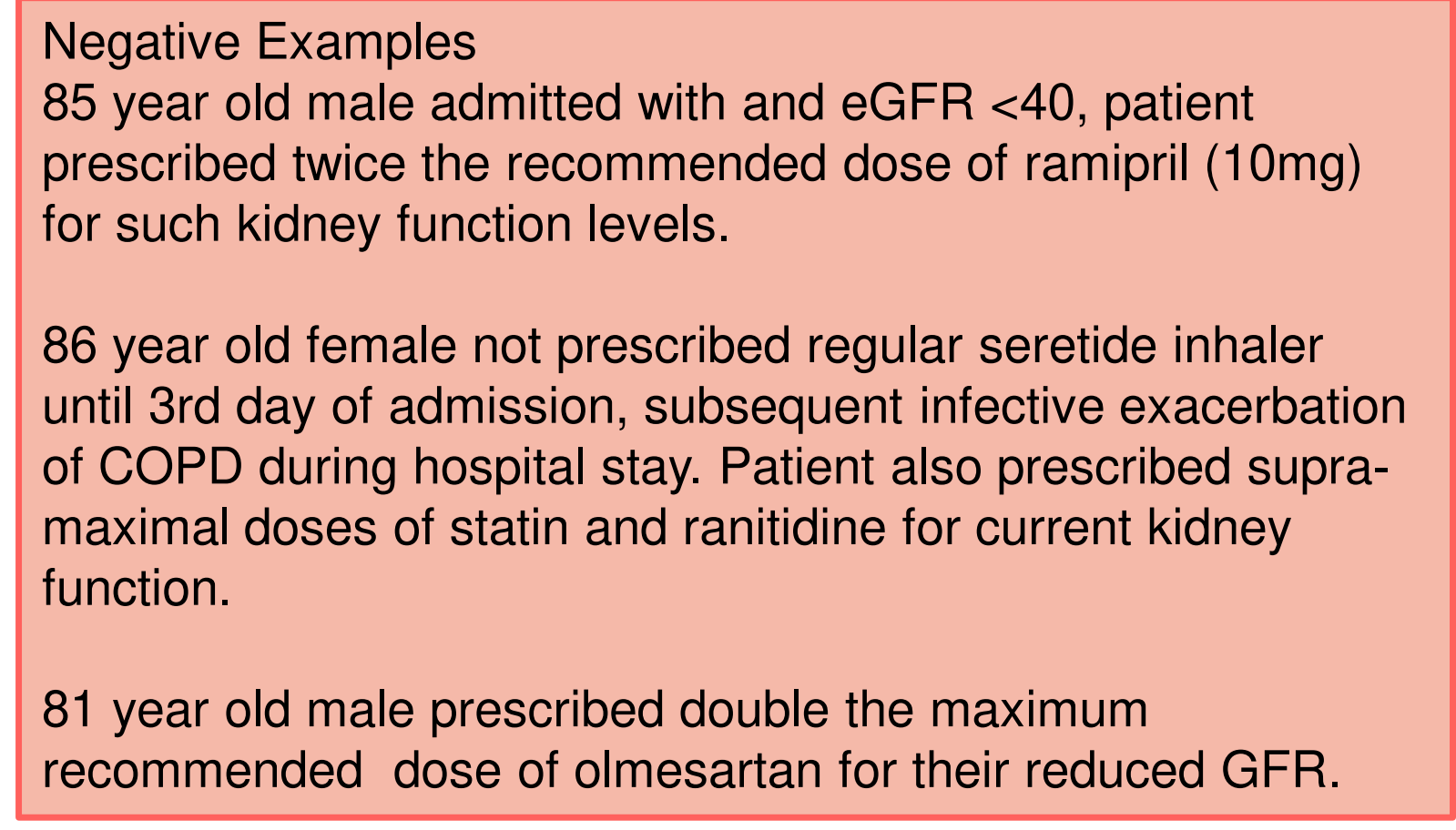

CKD in sample population

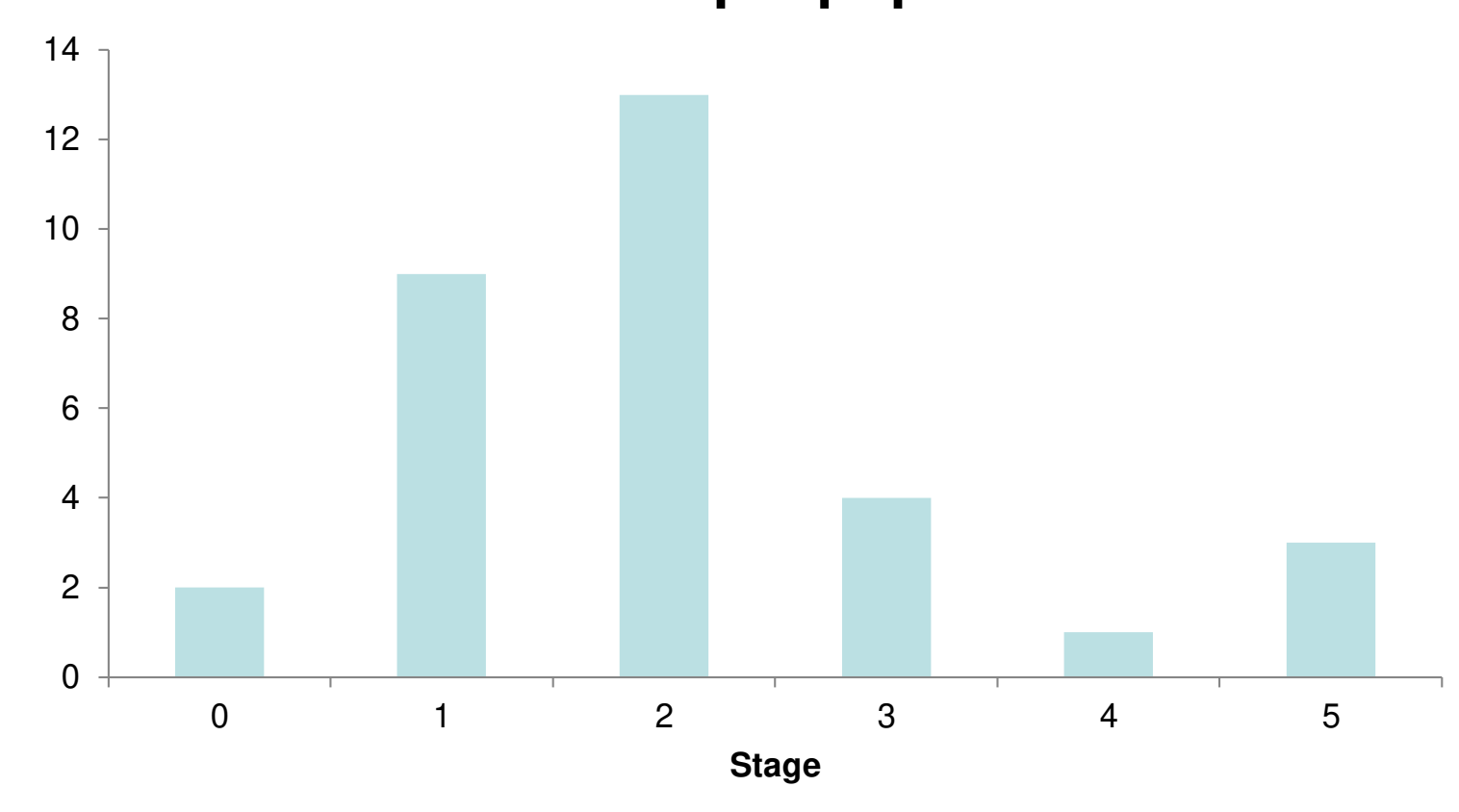

\section{Results and Discussion}

The Median age of patient investigated was 85 , and were prescribed on average 9 medications each. We found that a large proportion of patients were not prescribed their regular medications in the first 48hrs of admission. Of those patients that suffered a drop in GFR during admission, the minority had their medications altered accordingly and approximately half had the fluid maintenance appropriate for their renal function.

\section{Conclusion}

The study demonstrated a clear discontinuity of care between primary and secondary care, as well as a lack of recognition of the complex interactions that are likely to be inherent in such polypharmacy.

\section{References}

1 - 1 Mid-2015 Population Estimates UK Office for National Statistics, 2016

2 - Milton James C, Hill-Smith lan, Jackson Stephen H D. Prescribing for older people BMJ 2008; 336 :606 